\title{
Dimethyl fumarate attenuates experimental autoimmune neuritis through the nuclear factor erythroid-derived 2-related factor 2/ hemoxygenase-1 pathway by altering the balance of M1/M2 macrophages
}

Ranran Han, Jinting Xiao, Hui Zhai and Junwei Hao*

\begin{abstract}
Background: Guillain-Barré syndrome (GBS) is an acute, post-infectious, immune-mediated, demyelinating disease of peripheral nerves and nerve roots. Dimethyl fumarate (DMF), a fumaric acid ester, exhibits various biological activities, including multiple immunomodulatory and neuroprotective effects. However, the potential mechanism underlying the effect of DMF in GBS animal model experimental autoimmune neuritis (EAN) is unclear.

Methods: Using EAN, an established GBS model, we investigated the effect of DMF by assessing clinical score, histological staining and electrophysiological studies. Then, we further explored the potential mechanism by Western blot analysis, flow cytometry, fluorescence immunohistochemistry, PCR, and ELISA analysis. The MannWhitney $U$ test was used to compare differences between control group and treatment groups where appropriate.

Results: DMF treatment reduced the neurological deficits by ameliorating inflammatory cell infiltration and demyelination of sciatic nerves. In addition, DMF treatment decreased the level of pro-inflammatory M1 macrophages while increasing the number of anti-inflammatory M2 macrophages in the spleens and sciatic nerves of EAN rats. In RAW 264.7, a shift in macrophage polarization from M1 to M2 phenotype was demonstrated to be depended on DMF application. In sciatic nerves, DMF treatment elevated the level of the antioxidant transcription factor nuclear factor erythroid-derived 2-related factor 2 (Nrf2) and its target gene hemoxygenase-1 (HO-1) which could facilitate macrophage polarization toward M2 type. Moreover, DMF improved the inflammatory milieu in spleens of EAN rats, characterized by downregulation of messenger RNA (mRNA) of IFN- $\gamma$, TNF- $a$, IL- 6 , and IL-17 and upregulation of mRNA level of IL-4 and IL-10.
\end{abstract}

Conclusions: Taken together, our data demonstrate that DMF can effectively suppress EAN, and the mechanism involves altering the balance of M1/M2 macrophages and attenuating inflammation.

Keywords: Experimental autoimmune neuritis, Macrophages, Dimethyl fumarate, Guillain-Barré syndrome, Cytokine

\footnotetext{
* Correspondence: hjw@tmu.edu.cn

Department of Neurology, Tianjin Neurological Institute, Tianjin Medical

University General Hospital, Anshan Road, Heping District, Tianjin 300052,

China
} 


\section{Background}

Guillain-Barré syndrome (GBS), known as an acute inflammatory disorder in the peripheral nervous system (PNS), can cause rapidly progressive, symmetrical weakness of the extremities [1]. Proven effective immunotherapies for GBS are plasma exchange and intravenous treatment with immunoglobulin G [1]. Of note, approximately $5 \%$ of GBS patients die and up to $20 \%$ suffer from severe disability, irrespective of whether they receive immunotherapies [2]. Hence, further researches are imperative to improve this situation.

Experimental autoimmune neuritis (EAN), a wellknown animal model of GBS, effectively mimics clinical, histopathological, and electrophysiological features of GBS. EAN is characterized pathologically by a breakdown of the blood-nerve barrier, robust accumulation of reactive $\mathrm{T}$ cells and macrophages into the PNS, and demyelination of peripheral nerves [3]. Pro-inflammatory cytokines including IL-6, IFN- $\gamma$, IL-17, and TNF- $\alpha$, mostly secreted by Th1 and Th17 cells, predominate in sciatic nerves and lymphoid organs during the acute phase of GBS and contribute to inflammatory impairment of peripheral nerves, whereas anti-inflammatory cytokines such as IL-4 and IL-10, mainly secreted by Th2 cells, are proven beneficial for the protection of inflammatory impairment of peripheral nerves $[4,5]$.

The polarized macrophages are generally termed proinflammatory M1 or classically activated macrophages and anti-inflammatory M2 or alternatively activated macrophages [6, 7]. M1 macrophages, which arise mainly in response to IFN- $\gamma$ [8], are involved in the pathogenesis and course of EAN through their antibody-dependent cellular cytotoxicity (ADCC) and phagocytosis and myelin sheath damage [9]. M2 phenotype macrophages which arise mainly in response to IL-4 [8], however, contribute to recovery by promoting $\mathrm{T}$-cell apoptosis and the secretion of anti-inflammatory cytokines [10], promoting myelin repair and axonal regeneration [11]. Evidence shows that a switch in macrophage phenotype from M1 to M2 could influence the pathogenesis of autoimmune and inflammatory diseases [12]. We hypothesize that treatment facilitating the switch of macrophage phenotype from M1 to M2 may favor the outcome of EAN.

Dimethyl fumarate (DMF), an orally bioavailable fumaric acid ester, has been used successfully as a drug for treating the autoimmune disorders such as psoriasis since the 1990s [13] and relapsing/remitting multiple sclerosis (MS) since 2013 [14]. Recent report shows that it can ameliorate EAN as well [15], but the exact mechanisms underlying its effect is still unclear. In addition, previous studies demonstrate that DMF ameliorates MS by reducing inflammation $[16,17]$ and enhancing activation of the nuclear factor erythroid-derived 2-related factor 2 (Nrf2) antioxidant pathway [18]. As a basic leucine-zipper (bZip) transcription factor, Nrf2 protects a variety of tissues and cells against oxidative, inflammatory, and electrophilic stress through antioxidant response element (ARE)-mediated induction of diverse phase II detoxification and antioxidant enzymes, including $\mathrm{NAD}(\mathrm{P}) \mathrm{H}$ quinone oxidoreductase 1 (NQO1) and hemoxygenase-1 (HO-1) $[19,20]$. As the major antiinflammatory and anti-oxidative enzyme that is regulated by activating $\mathrm{Nrf2}$ [21], HO-1 induction has been reported to affect macrophage polarization toward M2 phenotype in experimental animal models of diabetes, Crohn's disease, hypertension, alcoholic liver disease, and intestinal injury [22]. Herein, we hypothesized that HO-1 induction by DMF may exert a protection against EAN.

The objective of this study was to assess the effect of DMF in treating EAN and the underlying mechanisms. DMF was applied in a preventative and a therapeutic paradigm, and the effect on clinical, histological, and immunological parameters was assessed. It is demonstrated that DMF is effective in treating EAN and the mechanism of action involves mobilization of anti-inflammatory and immunomodulatory responses mediated by the Nrf2/HO-1 pathway.

\section{Methods}

Experimental animals and group assignments

Male Lewis rats, 6-8 weeks old (160-190 g), were purchased from the Vital River Corporation (Beijing, China). All animals were acclimated to the vivarium environment and were maintained under temperature-controlled conditions and a 12-h light/dark cycle for 1 week. Food and water were provided ad libitum. Animals were randomly assigned to three groups (preventative, therapeutic, or control, $n=6$ per group) and each group was repeated 3 times. All efforts were made to minimize the number of animals used and any suffering they might experience. The experimental procedures were approved by the Animal Ethics Committee of the Tianjin Medical University.

\section{Induction and clinical evaluation of EAN}

EAN was induced by injecting both hind footpads with $300 \mu \mathrm{l}$ of an inoculum containing $300 \mu \mathrm{g}$ of dissolved P0 peptide 180-199 (10 mg/ml; Bio-Synthesis Corporation). The peptide was dissolved in phosphate-buffered saline (PBS) $(2 \mathrm{mg} / \mathrm{ml})$ and then emulsified with an equal volume of complete Freund's adjuvant (CFA; Difco) containing Mycobacterium tuberculosis (strain H37RA) to a final concentration of $1 \mathrm{mg} / \mathrm{ml}$. Following immunization, clinical signs of EAN were scored and quantified as follows: $0=$ normal; $1=$ reduced tonus of the tail; $2=\operatorname{limp}$ tail; $3=$ absent righting reflex; $4=$ gait ataxia; $5=$ mild paresis of the hind limbs; $6=$ moderate paraparesis; $7=$ 
severe paraparesis or paraplegia of the hind limbs; $8=$ tetraparesis; $9=$ moribund; and $10=$ death.

\section{DMF treatment}

Dimethyl fumarate (DMF; $97 \%$ pure) (Sigma-Aldrich) was freshly prepared in $0.08 \%$ carboxymethyl cellulose (CMC)/PBS. For preventative treatment, DMF solution was administered by oral gavage $(25 \mathrm{mg} / \mathrm{kg}$ body weight/ day) from day 1 to day 16 post-immunization (i.e., peak phase) or day 27 post-immunization (i.e., remission phase). For therapeutic treatment, DMF solution was administered by oral gavage at the same dose daily from the day on which the first clinical signs were observed, namely from day 7 to day 16 or 27 post-immunization. Control animals received the same volume of the vehicle solution (i.e., $0.08 \% \mathrm{CMC} / \mathrm{PBS}$ ). The dose of DMF was based on previously published studies [23, 24] and on preliminary studies done in our laboratory.

\section{Electrophysiological studies}

Electromyographic (EMG) recordings of the left sciatic nerve were made on day 16 post-immunization (i.e., peak phase) using a fully digital Keypoint Compact EMG/NCS/EP recording system (Dantec Co). A single blind trial method was used to record evoked compound muscle action potential (CMAP) amplitudes and latencies of sciatic nerves, as previously described [25]. Rats were anesthetized first with chloral hydrate (intraperitoneally, $3 \mathrm{mg} / \mathrm{kg}$ ). Two pairs of monopolar needle electrodes were used to stimulate the sciatic nerve and record the signals, respectively. After exposing the left sciatic nerve from the hip (proximal) to the ankle (distal), one pair of needle electrodes was inserted at the sciatic notch (hip/proximal) or the ankle (ankle/distal). The nerve stimulation parameters used to elicit CMAPs were $1-\mathrm{Hz}$ pulses, with each pulse being $5 \mathrm{~mA}$ in amplitude and $0.3 \mathrm{~ms}$ in duration. The recording electrodes were positioned in the "belly" part of the gastrocnemius muscle to record evoked potentials from stimulating the sciatic nerve. The motor nerve conduction velocity (MNCV) was calculated by measuring the distance between stimulating cathode electrodes and then measuring the latency difference. The amplitude was calculated from the baseline to the maximal peak under the resulting CMAP curves. After electrophysiologic measurements were completed, the incision was sutured under an aseptic environment. Body temperatures of rats during electrophysiologic measurements were maintained above $34{ }^{\circ} \mathrm{C}$ by positioning a heating pad under the rat. For each animal, triplicate measurements were made.

\section{Histopathological assessment}

Following nerve conduction studies, the sciatic nerves of each rat were harvested at the peak of disease (day 16 post- immunization). Six rats from each group received general anesthesia and were then perfused intracardially, first with $4{ }^{\circ} \mathrm{C}$ PBS for $2 \mathrm{~min}$, followed by $4 \%$ paraformaldehyde dissolved in PBS for $5 \mathrm{~min}$. The sciatic nerves were rapidly removed and fixed in $4 \%$ paraformaldehyde overnight at $4{ }^{\circ} \mathrm{C}$, and then the nerves were embedded in paraffin.

To evaluate the extent of mononuclear cell (MNC) infiltration and demyelination, serial transverse sections $(6 \mu \mathrm{m})$ were stained with hematoxylin-eosin (H\&E) (Solarbio Science \& Technology) and luxol fast blue (LFB), which contains $0.1 \%$ LFB solution, $0.1 \%$ Cresyl Echt Violet solution, and $0.05 \%$ lithium carbonate solution. Infiltrating inflammatory cells in H\&E-stained tissues were counted by image analysis using a Nikon Coolscope digital microscope (Nikon). Cell numbers were calculated per square millimeter from five random microscopic fields (200× magnification). All counts were performed in a blinded fashion. The average results were expressed as cells per square millimeter of tissue section. To evaluate the severity of demyelination, histological scores were calculated. Sections containing all perivascular areas were assessed by two independent observers (who were blinded to the treatment) according to the following semi-quantitative pathological/ histological scale: 0 , normal perivascular area; 1 , mild cellular infiltrate adjacent to the vessel; 2 , cellular infiltration plus demyelination in immediate proximity to the vessel; 3, cellular infiltration and demyelination throughout the section.

\section{Fluorescence immunohistochemistry}

Following nerve conduction studies, the sciatic nerves were harvested at the peak of disease (i.e., day 16 postimmunization) as described above and post-fixed in $4 \%$ paraformaldehyde overnight. The nerves were dehydrated sequentially in $15 \%$ and then $30 \%$ sucrose until the tissue equilibrated. After that, nerves were embedded in Tissue-tek medium (SAKURA) and snap-frozen in liquid nitrogen to expose antigenic sites for staining. Transverse sections $(8 \mu \mathrm{m})$ were made on a cryostat (Leica Microsystems LM3050S) and then mounted on poly-L-lysine-coated slides and stored at $-80{ }^{\circ} \mathrm{C}$.

Fluorescence immunohistochemistry of the frozen sections was performed using standard protocols provided by the antibody manufacturers. Briefly, after bringing the slides to room temperature for $20 \mathrm{~min}$, the mounted tissue was fixed in $4 \%$ paraformaldehyde for $10 \mathrm{~min}$ and permeabilized in $0.3 \%$ Triton X100 for $5 \mathrm{~min}$. Sections were washed in cold PBS after each step. After blocking with $3 \% \mathrm{BSA}$ for $30 \mathrm{~min}$ at $37{ }^{\circ} \mathrm{C}$, slides were incubated with primary antibodies: rabbit anti-Nrf2 (1:200, Abcam); rabbit anti-HO-1 (1:200, Abcam); mouse anti-CD68 (1:200, Abcam); goat anti-Iba1 (1:500, Abcam); rabbit anti-Iba1 (1:500, Wako); rabbit anti-iNOS (1:200, Abcam); rabbitCD86 (1:200, Abcam); goat anti-Arginase-1 (1:200, Santa 
Cruz Biotechnology, Inc); and goat anti-CD206 (1:200, Santa Cruz Biotechnology, Inc) at $4{ }^{\circ} \mathrm{C}$ overnight. The next day, they were washed in PBS and then incubated for $60 \mathrm{~min}$ at room temperature with the following speciesappropriate fluorochrome-conjugated secondary antibodies: Rhodamine (TRITC) AffiniPure donkey anti-goat IgG $(\mathrm{H}+\mathrm{L})(1: 100$, Jackson Immunoresearch); Rhodamine (TRITC)-conjugated AffiniPure goat anti-rabbit IgG ( $\mathrm{H}+$ L) (1:100, Jackson, Immunoresearch); Rhodamine (TRITC)conjugated AffiniPure goat anti-mouse IgG $(\mathrm{H}+\mathrm{L})(1: 100$, Jackson, Immunoresearch); Alexa Fluor 488 conjugated donkey anti-rabbit $\operatorname{IgG}(\mathrm{H}+\mathrm{L}) \quad(1: 1000$, Thermo Fisher Scientific); and Alexa Fluor 488 conjugated donkey antigoat IgG (H + L) (1:1000, Thermo Fisher Scientific). Finally, the slides were washed in PBS, and the tissue was coverslipped with fluoro-shield mounting medium containing DAPI (Abcam) to counterstain cell nuclei. Image analysis of stained cells of the entire area of each tissue section per sciatic nerve was performed using a Nikon Coolscope (Nikon). Positive cell numbers were calculated per square millimeter from three random microscopic fields (200× magnification). All counts were performed in a blinded fashion.

\section{Flow cytometry}

The polarization state of macrophages derived from rat spleens was determined at the peak of disease (i.e., day 16 post-immunization) by flow cytometric analysis. In brief, spleens were removed under aseptic conditions and splenocytes were harvested. For extracellular staining, $1 \times 10^{6}$ spleen MNCs were resuspended in $100 \mu \mathrm{lBS} / 1$ \% BSA and were co-stained for cell surface CD11b conjugated with phycoerythrin (1:100, Abcam), Ly6G conjugated with FITC (1:100, Abcam) and/or CD206 (1:100, Abcam) for $45 \mathrm{~min}$ at room temperature (RT) following manufacturer's specification. For intracellular staining, cells were first stained with CD11b and Ly6G as above, then fixed with Fixation Buffer (BioLegend), permeabilized with Permeabilization Wash Buffer (BioLegend), and then stained with a primary antibody for intracellular iNOS (1:100, Abcam) and Alexa Fluor 647 -labelled donkey antirabbit IgG (H\&L) secondary antibody (1:100, Abcam). MNCs were gated by forward and sideward scatter. Surface and intracellular molecule expression were assessed by determining the positive cell percentage. Cells from all groups were collected and analyzed at each time point on the same day with the same cytometer settings. Flow cytometric data were acquired using a FACSAria ${ }^{\text {Tu }}$ flow cytometer (BD Biosciences) and analyzed with FlowJo software version 7.6.1 (flowjo.com).

\section{Western blot analysis}

After removal, part of the sciatic nerves were immediately stored in liquid nitrogen until needed for protein isolation by TRIzol (Ambion). All procedures were performed following manufacturer's introduction for obtaining total protein. After boiling, samples were adjusted to equal content and volume before being loaded on $10 \%$ SDS-PAGE gels and electrophoretically separated. Proteins were then transferred to PVDF membranes (Millipore) followed by blocking. Proteins were visualized by using primary antibodies for Nrf2 (1:1000, Abcam) and HO-1 (1:2000, Abcam), actin (1:1000, Zhongshanjinqiao), and then by incubating with goat anti-rabbit horseradish peroxidaseconjugated secondary antibody (1:5000, TRANSGENE BIOTECH) and goat anti-mouse secondary antibody (1:5000, TRANSGENE BIOTECH). The protein-specific signals were detected using a Bio-Rad 721BR08844 Gel Doc Imager (Bio-Rad).

\section{Lymphocyte proliferation assay}

Antigen-specific lymphocyte proliferation was measured by MTS (3-(4, 5-dimethylthiazol-2-yl)-5-(3-carboxymethoxyphenyl)-2-(4-sulfophenyl)-2H-tetrazolium) assays on day 16 post-immunization as described previously [26]. Briefly, spleens were removed under aseptic conditions and splenocytes were harvested. Single-cell suspensions of MNCs at a density of $2 \times 10^{6}$ cells $/ \mathrm{ml}$ were allocated with $100-\mu \mathrm{l} \mathrm{cul}-$ ture medium into 96-well microtiter plates. The culture medium was RPMI1640 (containing $2.05 \mathrm{mM}$ glutamine; HyClone) supplemented with $1 \%(v / v)$ HEPES buffer solution (Gibco), $0.1 \%(v / v)$ 2-mercaptoethanol (Gibco), $1 \%$ $(v / v)$ sodium pyruvate (Gibco), and $1 \%(v / v)$ Pen Strep (Gibco), and $10 \%(v / v)$ fetal bovine serum (FBS; Gibco). MNCs were cultured in the presence of either P0 peptide 180-199 (10 $\mu \mathrm{g} / \mathrm{ml}$; Bio-Synthesis Corporation) or without antigen. The concentration of the peptide used was based on previous studies [26, 27]. Following incubation for $72 \mathrm{~h}$ at $37{ }^{\circ} \mathrm{C}$ and $5 \% \mathrm{CO}_{2}$, MTS solution (Promega) was added and cells were incubated for an additional $4 \mathrm{~h}$. The absorbance was measured at $490 \mathrm{~nm}$ using a microtiter plate reader (Titertek). Since we administered the drug in vivo, we could not calculate an inhibitory rate. The data are presented as mean $\mathrm{OD} \pm \mathrm{SEM}$. The experiment was performed with MNCs from four different rats in each group, and MNCs from each rat were done in triplicate.

Real-time quantitative polymerase chain reaction (RT-PCR) Spleens were removed under aseptic conditions and splenocytes were harvested as described above. $10^{7}$ splenic cells were stored for proceduring RNA isolation by TRIzol (Ambion). All actions were performanced following instruction book to get total RNA (10 $\mu \mathrm{g} / \mathrm{sample})$. Total RNA was reverse transcribed into complementary DNA (cDNA) using TransScript First-Strand cDNA Synthesis SuperMix (TRANSGEN BIOTECH). All the procedures were strictly performed as per instructions. The primers used to measure gene expression are the following: 
IFN- $\gamma$ (sense, TCGCACCTGATCACTAACTTCTTC; antisense, CGACTCCTTTTCCGCTTCC), TNF- $\alpha$ (sense, TGA TCG GTC CCA ACA AGG A; antisense, TGC TTG GTG GTT TGC TAC GA), IL-4 (sense, TGA TGG GTC TCA GCC CCC ACC TTG C; antisense, CTT TCA GTG TTG TGA GCG TGG ACT C), IL-17 (sense, TGGACTCTGA GCCGCATTGA; antisense, GACGCATGGCGGACAATA GA),IL-4 (sense, TGATGGGTCTCAGCCCCCACCTTGC; antisense, CTTTCAGTGTTGTGAGCGTGGACTC), $\beta-$ actin (sense, CCGTCTTCCCCTCCATCGT; antisense, AT CGTCCCAGTTGGTTACAATGC). The PCR program was run at $95^{\circ} \mathrm{C}$ for $10 \mathrm{~min}$ then 40 cycles at $95^{\circ} \mathrm{C}$ for $15 \mathrm{~s}$ and $60{ }^{\circ} \mathrm{C}$ for $1 \mathrm{~min}$. The results were automatically analyzed by the ABI Stepone Plus instrument, and the method of $2^{-\Delta \Delta \mathrm{Ct}}$ $(\Delta \mathrm{Ct}$ represents the difference of threshold cycle value between the target gene and the inner control; $\Delta \Delta \mathrm{Ct}$ represents the difference of $\Delta \mathrm{Ct}$ between different groups) was used to analyze the messenger RNAs (mRNA) expression. Results were calculated as levels of target mRNAs relative to $\beta$-actin (four samples from each group were analyzed by PCR).

\section{ELISA for cytokine profile}

The supernatants of splenic MNCs $\left(2 \times 10^{6}\right.$ cells $\left./ \mathrm{ml}\right)$ cultured with P0 peptide 180-199 $(10 \mu \mathrm{g} / \mathrm{ml})$ were collected after incubation for $72 \mathrm{~h}$ at $37{ }^{\circ} \mathrm{C}$ and $5 \% \mathrm{CO}_{2}$. Simultaneous quantitative analysis of six cytokines, including IL-1 $\alpha$, IL-4, IL-6, IL-10, IFN- $\gamma$, TNF- $\alpha$, was performed using a multi-analyte ELISArray Kit (QIAGEN) according to the manufacturer's instructions. Determinations were performed in triple, and results are expressed as mean $\mathrm{OD} \pm \operatorname{SEM}(n=4)$.

\section{In vitro cell culture}

To further investigate the effects of DMF on macrophage polarization, we used immortalized murine macrophage cell line RAW 264.7 in vitro. RAW 264.7 cells were grown in complete RPMI 1640 medium (Life Technologies) containing penicillin $(100 \mathrm{U} / \mathrm{ml})$, streptomycin $(100 \mathrm{U} / \mathrm{ml})$, and $10 \%$ FBS (Gibco), maintained at $37{ }^{\circ} \mathrm{C}$ and $5 \% \mathrm{CO}_{2}$. Briefly, $2 \times 10^{5}$ cells were seeded into 24 -well cell culture plates, triplicated either on coverslips pretreated with polylysine or not, and cultured for $24 \mathrm{~h}$. Then, cells were stimulated with LPS (Sigma-Aldrich) for $24 \mathrm{~h}$, and afterwards DMF $(0,20,50,100 \mu \mathrm{M})$ was added and incubated for another $24 \mathrm{~h}$. Thereafter, cells on slides were prepared for immunocytochemistry staining and the other cultured cells were harvested and centrifuged for RNA analyses (see below). The staining procedure of cells was similar to that of the tissue slides as mentioned above. Immunocytochemistry of macrophages on the slides used the following primary antibodies. To detect M1 macrophage markers, we used rabbit antibody to iNOS (1:200, Abcam). To detect M2 markers, we used goat antibody to Arginase-1
(1:200, Santa Cruz Biotechnology, Inc). Antibodies used to mark pan macrophage markers included mouse antibody to goat anti-Iba1 (1:500, Abcam); rabbit anti-Iba1 (1:500, Wako). Species-appropriate fluorochrome-conjugated secondary antibodies were added. Cells were observed by using fluorescence microscopy (Nikon). Five different fields of each slide were acquired; the pictures of each group were collected from triple-cultured cells. Fluorescence intensity of iNOS and Arginase-1 (Arg1) was analyzed semiquantitatively by image analysis software (Image ProPlus 6.0 Software). Results were provided as relative intensity comparing DMF-treated cells to PBS-treated cells.

Total RNA from cultured cells was prepared as described above according to the manufacturer's instructions. Total RNA was reverse transcribed into cDNA using TransScript First-Strand cDNA Synthesis SuperMix (TRANSGEN BIOTECH). The mRNA expression levels were measured by real-time PCR using the following primers: $\beta$-actin (sense, TGG AAT CCT GTG GCA TCC ATG AAA; antisense, TAA AAC GCA GCT CAG TAA CAG TCC G); TNF- $\alpha$ (sense, AAC TAG TGG TGC CAG CCG AT; antisense, CTT CAC AGA GCA ATG ACT CC); iNOS (sense, CAG CTG GGC TGT ACA AAC CTT; antisense, CAT TGG AAG TGA AGC GTT TCG); Arg 1 (sense, TTA GGC CAA GGT GCT TGC TGC C; antisense, TAC CAT GGC CCT GAG GAG GTT C); and IL-10 (sense, TCA TTC ATG GCC TTG TAG ACA C; antisense, AGC TGG ACA ACA TAC TGC TAA C). The PCR program was run at $95{ }^{\circ} \mathrm{C}$ for $10 \mathrm{~min}$, then 40 cycles at $95{ }^{\circ} \mathrm{C}$ for $15 \mathrm{~s}$, and finally at $60{ }^{\circ} \mathrm{C}$ for $1 \mathrm{~min}$. The results were automatically analyzed by an ABI SteponePlus instrument, and the method of $2^{-\Delta \Delta C t}$ was used to analyze mRNA expression. Results were calculated as levels of target mRNAs relative to those from untreated RAW 264.7 cells. Four samples from each group were analyzed by PCR.

\section{Evaluation and statistical analysis}

The Mann-Whitney $U$ test was used to compare differences between control group and treatment groups where appropriate (GraphPad Prism 5.0). Data are presented as means \pm SEM. For all statistical analyses, the level of significance was set at $p<0.05$.

\section{Results}

DMF treatment attenuates clinical severity, histological changes, and inflammatory cell accumulation in EAN

Treatment of EAN in our rat model with DMF was investigated in two different paradigms (Fig. 1). In the preventative paradigm (blue line), we administered vehicle or DMF $(25 \mathrm{mg} / \mathrm{kg} /$ day) by gavage after immunization. In the therapeutic paradigm (red line), the treatment was started when the first clinical symptoms occurred on day 7 post-immunization. The severity of EAN was reduced in both the preventative and therapeutic treatment groups 


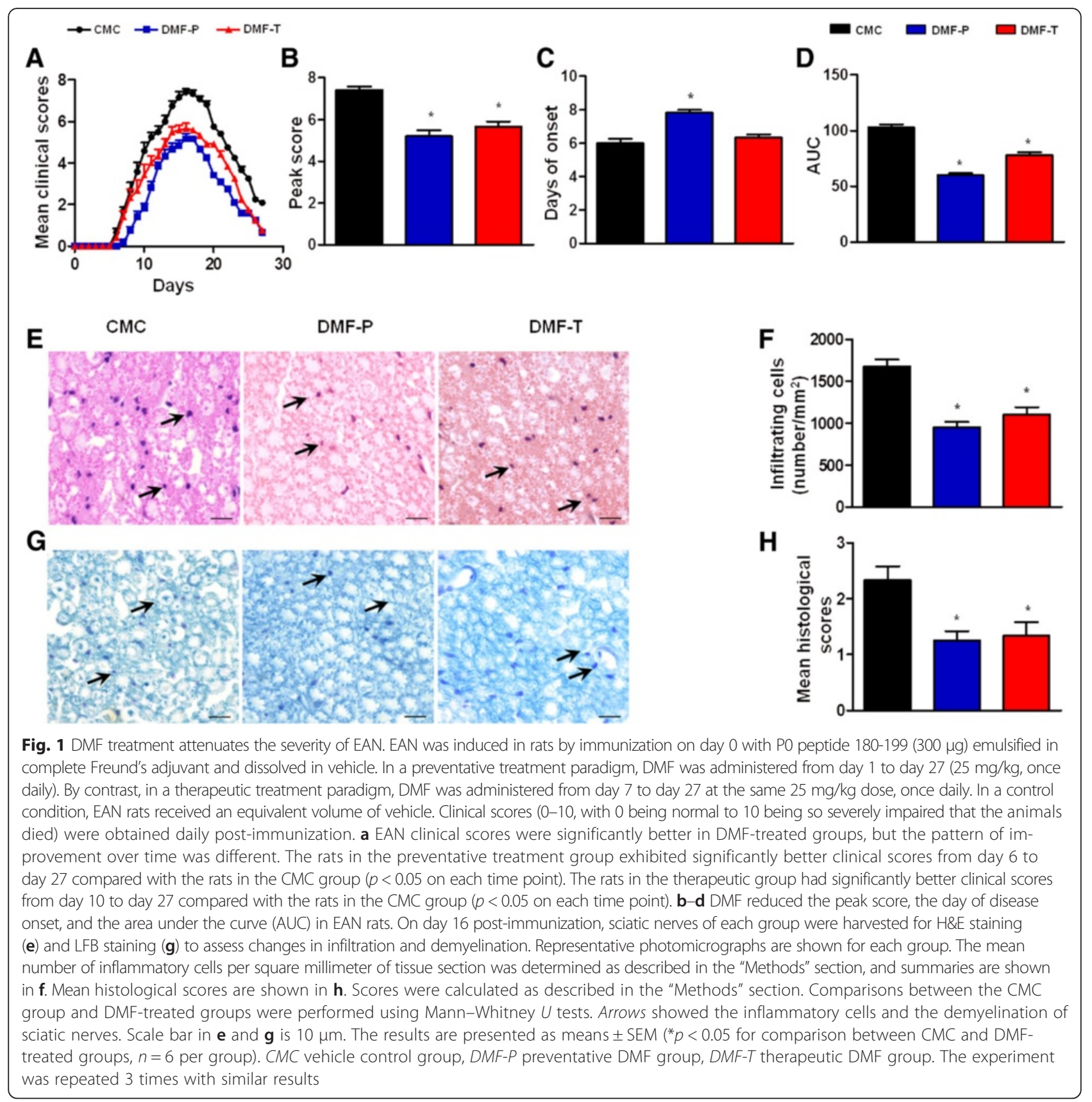

compared to the control group (CMC), as measured by clinical severity scores. The rats in the preventative treatment group exhibited significantly better clinical scores from day 6 to day 27 compared with the rats in CMC group ( $p<0.05$ on each time point). By contrast, clinical improvement of rats in the therapeutic group was delayed, with their clinical scores being lower than that of the rats in CMC group from day 8 to day 27 and the difference was significant only from day 10 to day $27(p<0.05$ on each time point). In addition, the mean peak clinical score was $5.20 \pm 0.30,5.67 \pm 0.25$, and $7.42 \pm 0.15$ in the preventative, therapeutic, and $\mathrm{CMC}$ groups, respectively
(Fig. 1a, b). The preventative treatment group delayed the onset of the first clinical sign of impairment (Fig. 1a, c). The day of EAN onset was day $7.83 \pm 0.17$ in the preventative group, $6.33 \pm 0.21$ in the therapeutic group, and 6.00 \pm 0.26 in the CMC group (Fig. 1c). DMF also reduced the AUC of the motor deficit assessed by clinical scoring (Fig. 1d). Overall, both preventative and therapeutic DMF treatment ameliorated EAN.

At the maximum of the clinical course of EAN (i.e., day 16 post-immunization), we evaluated the histopathology of the rats' sciatic nerves. H\&E staining was used to show inflammatory cell infiltration, and LFB staining was used to 
show myelin demyelination. Treating EAN rats with DMF in both treatment paradigms lowered the number of inflammatory cells (Fig. 1e, f). The incidence of demyelination and inflammatory cell infiltration was reduced by DMF treatment in both paradigms (Fig. 1g, h). When the stained tissue was semi-quantitatively graded, the mean histological scores were markedly higher in the $\mathrm{CMC}$ group $(2.33 \pm 0.25)$ compared to the scores of preventative $(1.25 \pm$ $0.17, p<0.05)$ and therapeutic $(1.33 \pm 0.25, p<0.05)$ groups (Fig. 1h).

DMF protects against EAN-induced peripheral nerve injury The ability of DMF to protect against EAN-induced peripheral nerve injury was assessed by evoked response electrophysiology at day 16 post-immunization in the sciatic nerve. It is well known that EAN rats exhibit decreasing CMAP amplitude and MNCV and lengthening of CMAP latency. In agreement with the clinical findings shown in Fig. 1, DMF prevented the development of peripheral nerve deficits in both DMF-treated groups (Fig. 2). For the MNCV, the CMC group's mean conduction velocity was slower than the preventative and therapeutic groups' conduction velocity $(37.73 \pm 3.49$ vs. $70.08 \pm 8.25 \mathrm{~m} / \mathrm{s}$ and $69.24 \pm 11.73 \mathrm{~m} / \mathrm{s}, p<0.05$, Fig. 2c). For CMAP latency, the preventative group and therapeutic group also showed a beneficial effect of DMF on CMAP latency, which was shorter than the CMC group's average latency $(0.29 \pm 0.03$ and $0.29 \pm 0.04 \mathrm{~ms}$ vs. $0.55 \pm 0.04 \mathrm{~ms}, p<0.05$, Fig. $2 \mathrm{~d}$ ). Also, the CMAP mean amplitudes of the two DMF treatment groups were greater $(12.25 \pm 2.52$ and $17.72 \pm 3.03 \mathrm{mV}$, respectively, Fig. 2e) compared to the CMC group $(10.25 \pm 2.04 \mathrm{mV})$, but this was not significant.

\section{DMF promotes M2 macrophage polarization in EAN rats and in vitro}

To further investigate the mechanism of DMF's beneficial effect in EAN, we assessed possible DMF-related changes in macrophage polarization in sciatic nerves and in spleens of EAN rats. Double-immunohistochemical staining was used to identify the proportion of M1 and M2 cells in sciatic nerves. Flow cytometry was also used to investigate polarization state of MNCs derived from spleens of EAN rats treated in the different DMF paradigms. In sciatic nerves harvested on day 16 post-immunization, DMFpreventative and DMF-therapeutic groups both displayed elevated local expression of M2 macrophages and reduced expression of M1 macrophages compared to the CMC group (Fig. 3a-c, $p<0.05$ and Additional file 1: Figure S1). Flow cytometry results of spleen MNCs followed the same

A

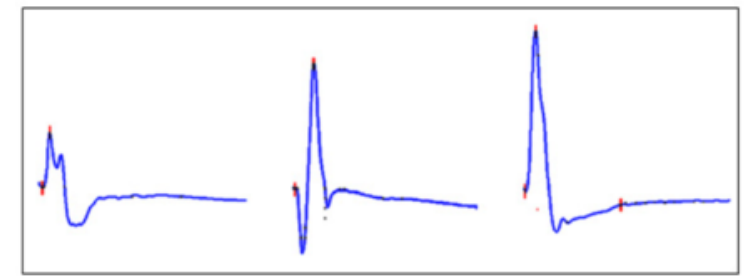

B
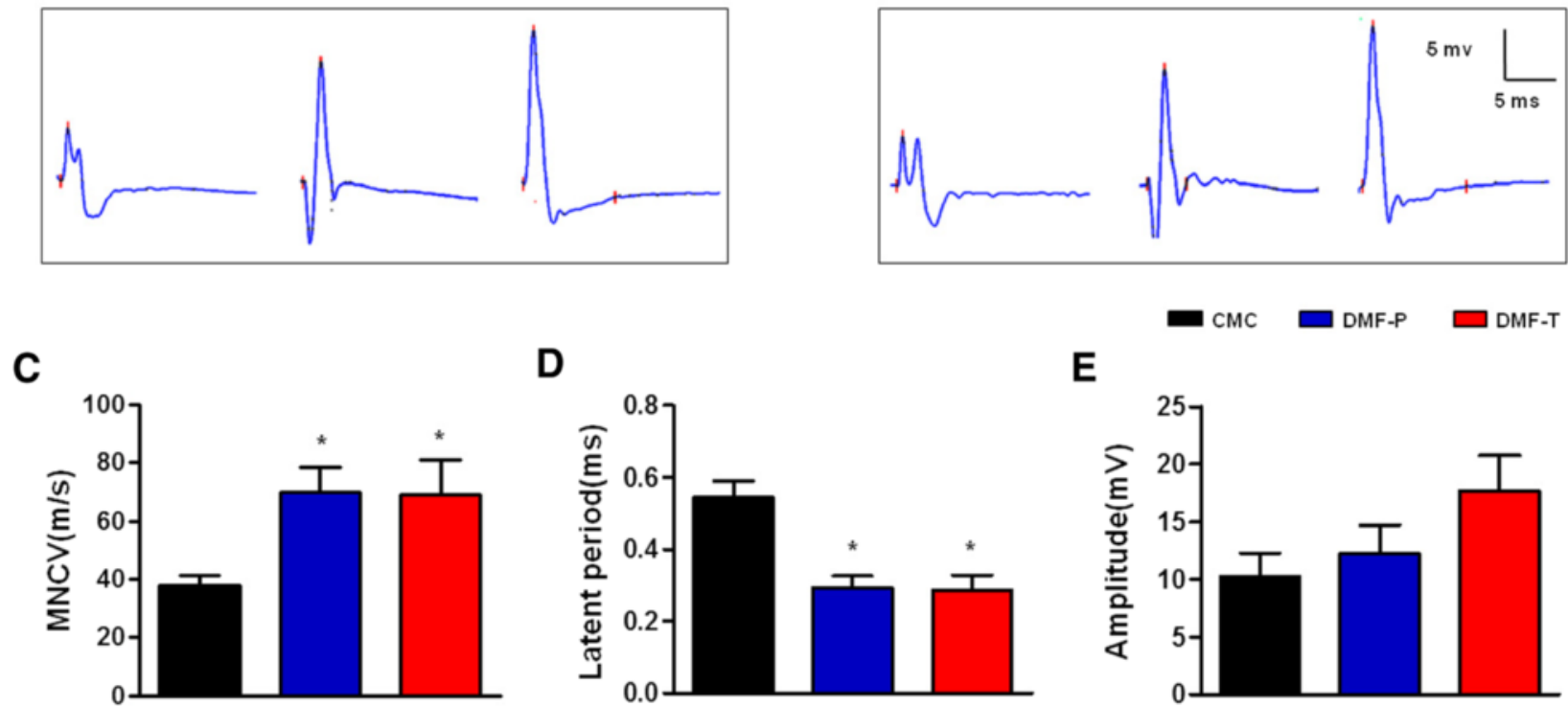

D

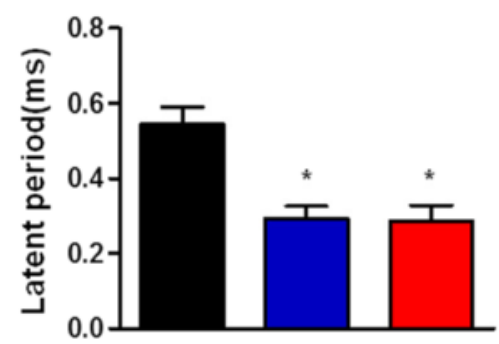

E
DMF-P

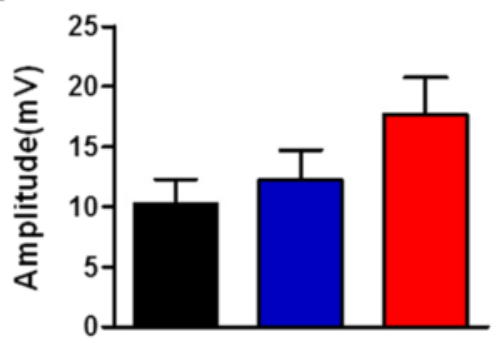

Fig. 2 DMF protects against peripheral nerve conduction deficits in EAN rats. Representative electrophysiological recordings of motor nerve compound muscle action potentials (CMAPs) evoked from stimulation of the fibular head (a) or ankle regions (b) of the sciatic nerve are shown for CMC, DMF-P, and DMF-T rats, respectively, from left to right. c Compared to the CMC group, DMF-treated groups were protected from EAN-induced damages of mean motor nerve conduction velocity (MNCV). $\mathbf{d}$ Compared to the CMC group, DMF-treated groups also exhibited better distal motor latencies of CMAPs. e DMF treatment improved the amplitude of CMAPs when compared to CMC groups, while we observed a non-significant trend. Comparisons between the CMC group and DMF-treated groups were performed using Mann-Whitney $U$ tests. Data shown are the means \pm SEM $\left({ }^{*} p<0.05\right.$ for comparison between CMC and DMF-treated groups; $n=5-6)$. CMC vehicle control group, DMF-P preventative DMF group, DMF-T therapeutic DMF group. The experiment was repeated 3 times with similar results 


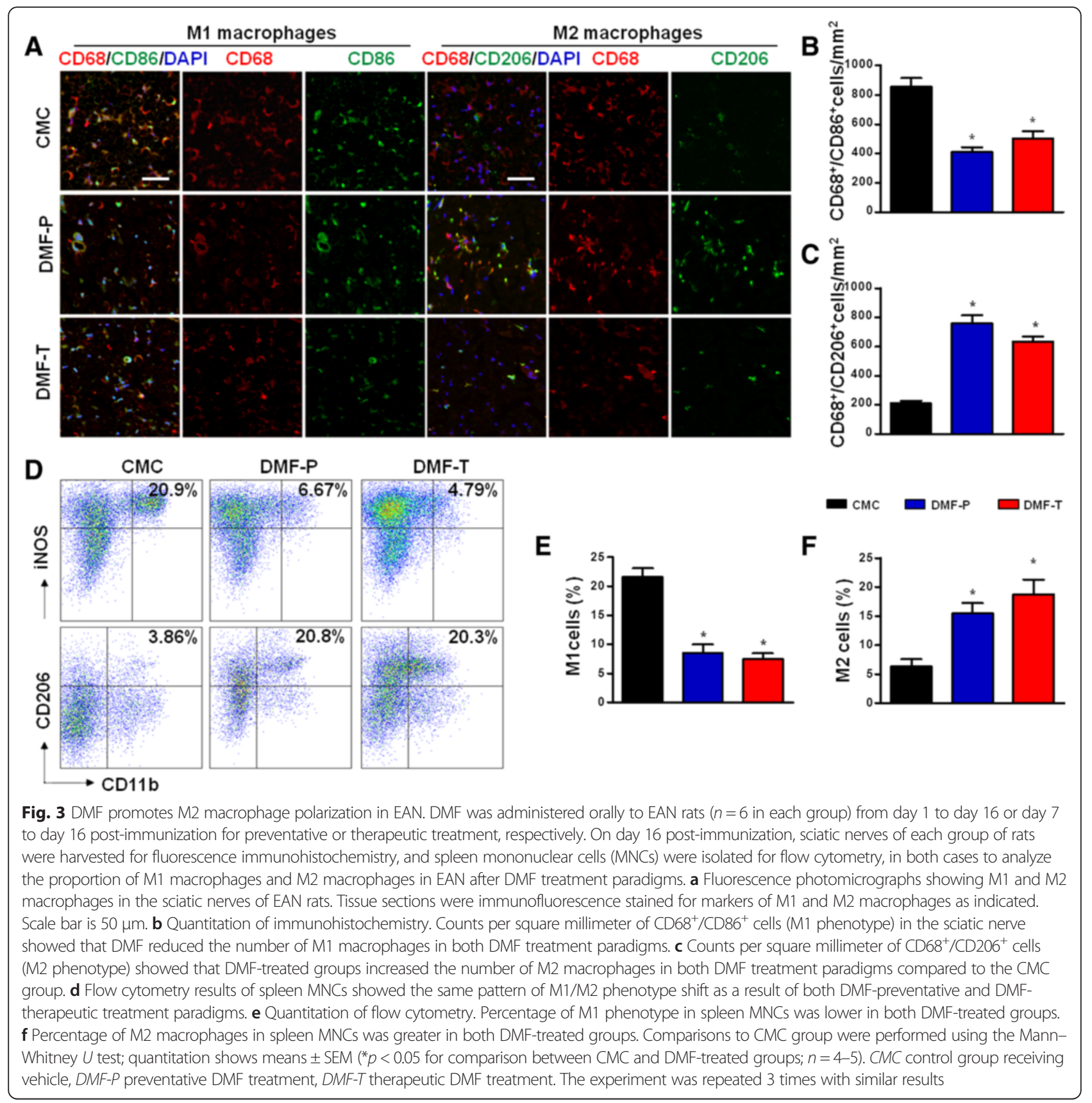

group patterns of M1/M2 expression. Both the DMFpreventative and DMF-therapeutic groups showed elevated expression of M2 and reduced expression of M1 macrophages in EAN spleens compared to the CMC group (Fig. 3d-f, $p<0.05$ ).

As a third line of evidence, we assessed the in vitro polarization shift of macrophages after DMF treatment using the murine macrophage cell line, RAW 264.7. The M1 macrophage phenotype was first induced by in vitro LPS application $(5 \mu \mathrm{g} / \mathrm{ml})$ for $24 \mathrm{~h}$. Various concentrations of DMF $(20,50$, and $100 \mu \mathrm{M})$ were then added to the culture, and the cells were incubated for another $24 \mathrm{~h}$. As shown in Fig. 4, in vitro DMF application reduced the expression of iNOS and increased the expression of Arg1 significantly (Fig. $4 \mathrm{a}-\mathrm{d}, p<0.05$ ) detected by fluorescence immunohistochemistry. DMF application also attenuated iNOS and TNF- $\alpha$ mRNA expression and increased Arg1 and IL-10 mRNA expression (Fig. $4 \mathrm{e}-\mathrm{h}, p<0.05$ ). These results suggested a shift in polarization from the M1 to M2 phenotype. Therefore, we verified in vitro that DMF can promote a shift to M2 polarization, and together with our previous results, these results showed that the shift can possibly be induced in vivo as well by DMF treatment. 


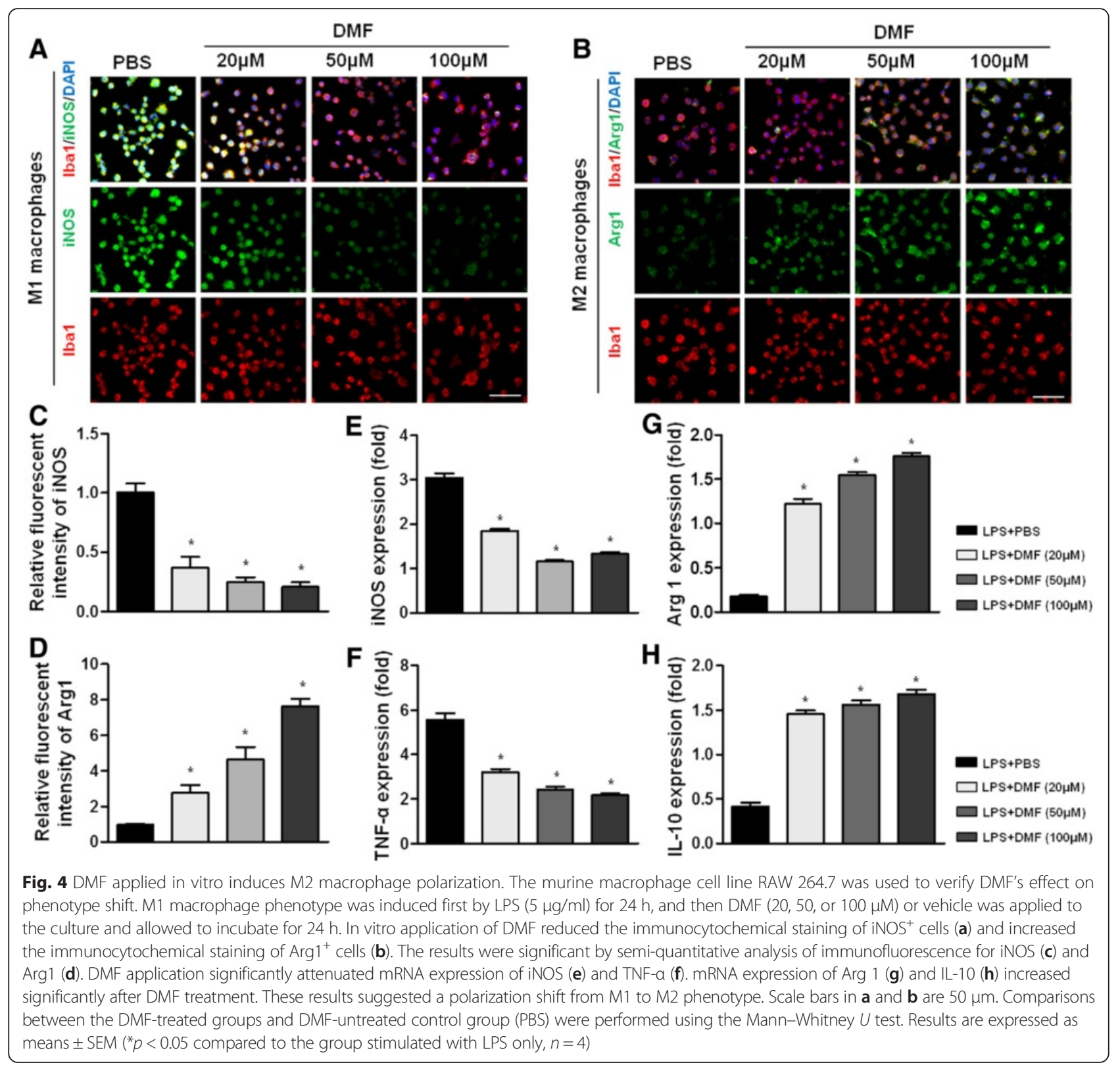

DMF increases Nrf2 and HO-1 protein levels in sciatic nerves of EAN rats

Nrf2 protein regulates the expression of antioxidant proteins that protect against oxidative damage related to inflammation, and HO- 1 is a key downstream mediator of Nrf2. As M2 macrophages highly express HO-1 [22], we explored the effect of DMF on the level of Nrf2 and HO-1. Sciatic nerves were taken from EAN rats on post-immunization day 16 for fluorescence immunohistochemistry and Western blotting analysis to study the cellular expression of $\mathrm{Nrf} 2 / \mathrm{HO}-1$. In sciatic nerve tissue sections, DMF-preventative and DMFtherapeutic groups showed robust localized cellular expression of $\mathrm{Nrf} 2$ and $\mathrm{HO}-1$ compared to the CMC group
(Fig. 5a, b, d, $p<0.05$ ). Furthermore, detection by Western blotting also showed an increased level of Nrf2 and HO-1 (Fig. 5e-g, $p<0.05$ ). In addition, co-staining macrophages with Nrf2 showed that DMF-treated groups had a higher radio of Nrf2-positive macrophages than CMC group (Fig. 5a, c, $p<0.05$ ).

DMF ameliorates lymphocyte proliferative responses and alters cytokine profile

MNCs prepared from EAN rat spleens on day 16 postimmunization were stimulated in vitro with P0 peptide $180-199(10 \mu \mathrm{g} / \mathrm{ml})$ or without peptide for $72 \mathrm{~h}$ to evaluate the effect of DMF on lymphocyte-proliferative responses (Fig. 6a). When compared to the CMC group, a significant 


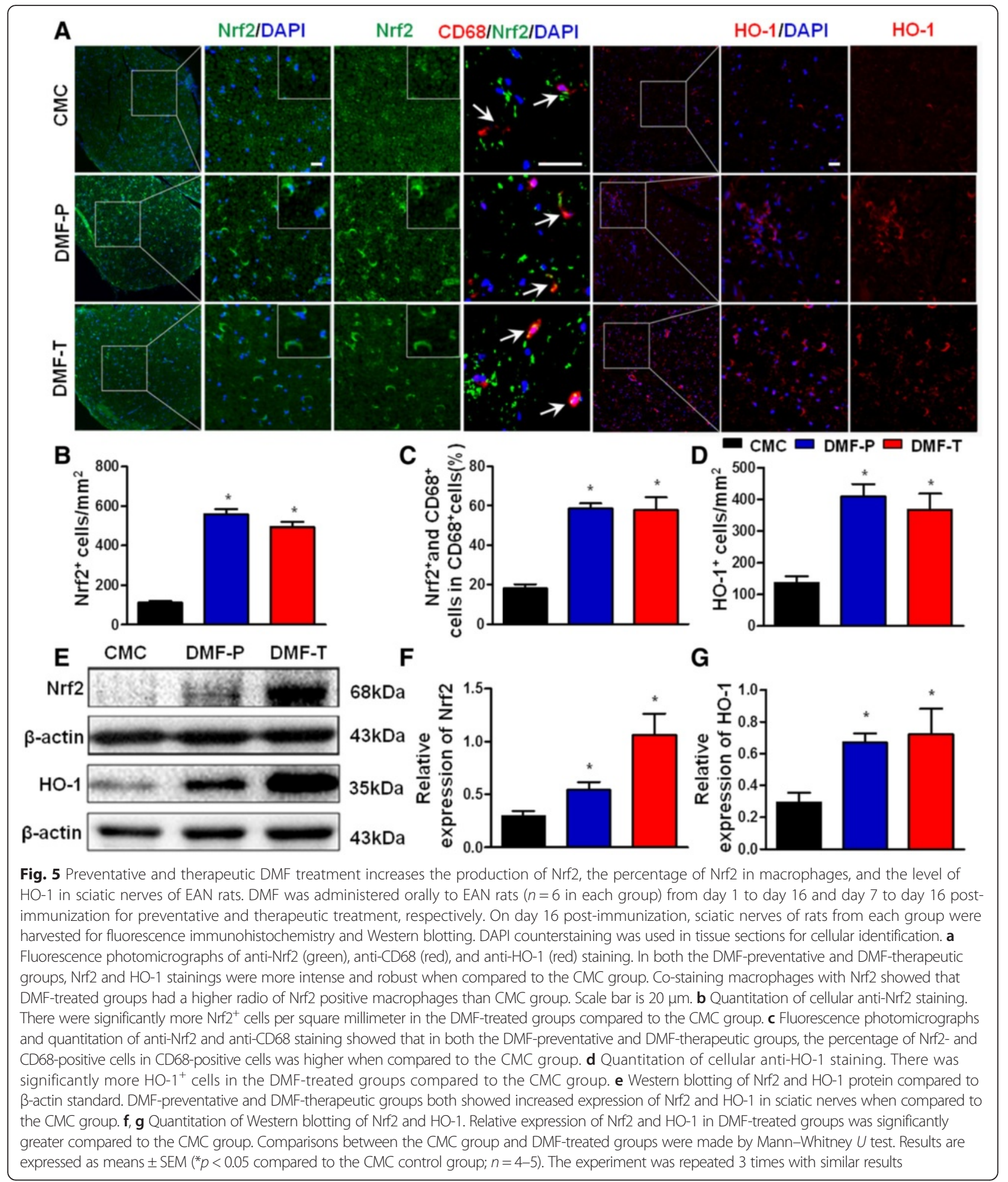

reduction in lymphocyte proliferation was observed in the preventative group and therapeutic group $(0.91 \pm 0.08$ vs. $0.58 \pm 0.02$, and $0.54 \pm 0.05$, respectively) (Fig. 6a, $p<$ 0.05 ), as measured by MTS assay with P0 peptide stimulation. The same pattern of lymphocyte proliferation was observed without peptide stimulation, and there was no significant difference between DMF treatment groups and the control group (data not shown).

As is well documented, the expression profiles of cytokines affect the outcome of EAN. Therefore, we semi- 

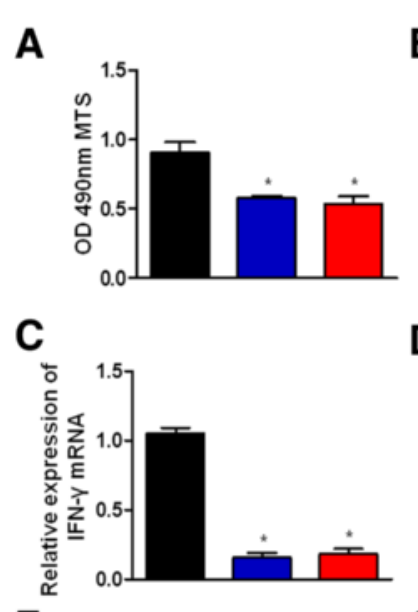

$\mathbf{F}$

B

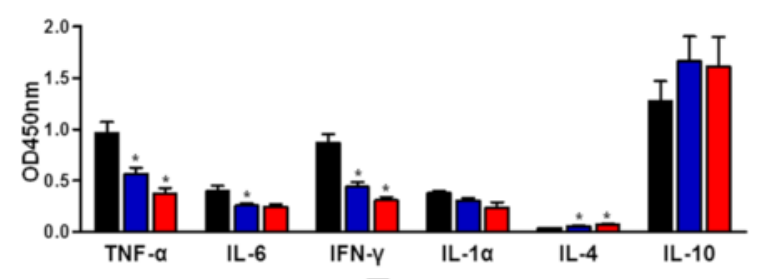

D

E
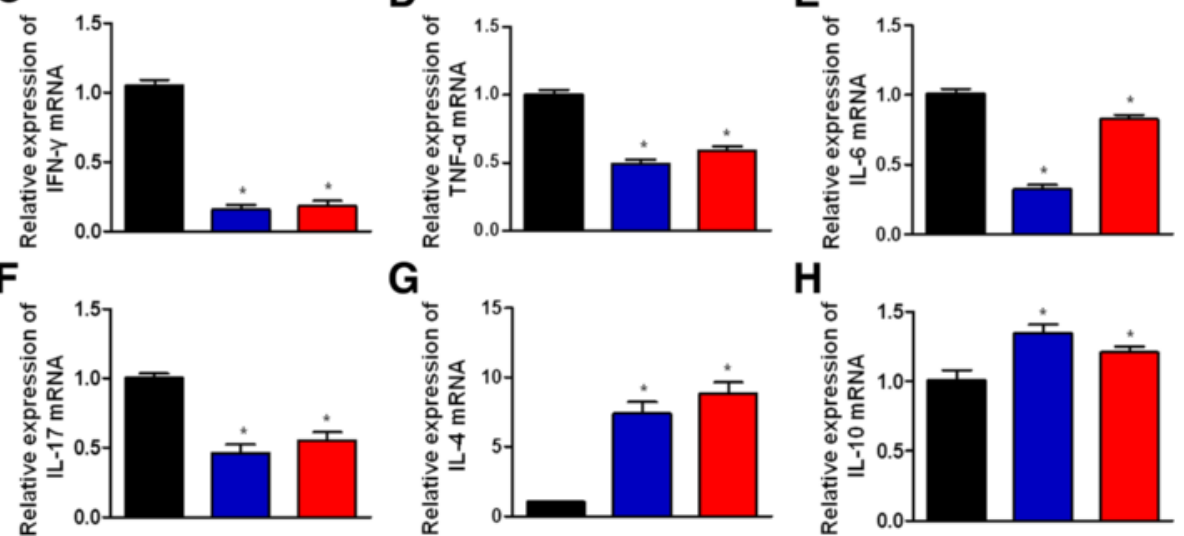

H

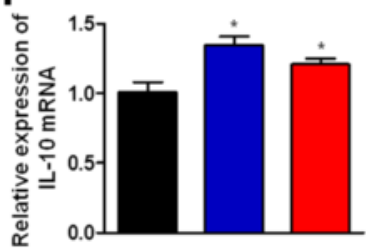

Fig. 6 DMF ameliorates lymphocyte proliferative responses and alters cytokine profiles. Splenic mononuclear cells (MNCs) from each group $(n=6)$ were harvested on day 16 post-immunization. a MNCs proliferation following DMF treatment. After culturing MNCs for $72 \mathrm{~h}$ in the presence of P0 peptide 180-199 (10 $\mathrm{\mu g} / \mathrm{ml})$, MNC proliferation was analyzed by MTS assay. When compared to the CMC group, a significant reduction in proliferation was observed in DMF-preventative and DMF-therapeutic groups. b Cytokine profiles of the supernatants of spleen MNCs cultured with P0 peptide $180-199$ (10 $\mu \mathrm{gg} / \mathrm{ml})$ for $72 \mathrm{~h}$, as measured by ELISA. The levels of TNF- $a$, IL-6, and IFN- $\gamma$ decreased greatly, but the level of IL-4 increased compared to the CMC group. A trend in increasing IL-10 levels and decreasing IL-1a levels was evident but not statistically significant. c-h IL-6, IL-4, IL-10, IFN- $\gamma$, TNF-a, and IL-17 mRNA expression levels in the splenocytes of EAN rats, as measured by RT-PCR. The level of pro-inflammatory cytokines IFN- $\gamma$, TNF- $a$, IL-6, and IL-17 decreased in DMF-treated groups, while the level of anti-inflammatory cytokines IL-4 and IL-10 increased significantly. Each experiment was performed in triplicate for each group. Comparisons between the CMC group and DMF-treated groups were performed using Mann-Whitney U tests. Data shown are means \pm SEM $\left({ }^{*} p<0.05\right.$ for comparison between CMC and DMF-treated groups, $\left.n=4\right)$. The experiment was repeated 3 times with similar results

quantitatively analyzed the effects of DMF treatment on the cytokine profiles produced in vitro by MNCs from EAN rats in response to the presence of P0 peptide 180199 using multi-cytokine ELISA kits. As shown in Fig. $6 \mathrm{~b}$, the production of pro-inflammatory cytokines (IL-6, IFN- $\gamma$, IL-1 $\alpha$, and TNF- $\alpha$ ) by MNCs cultured with P0 peptide $180-199(10 \mu \mathrm{g} / \mathrm{ml})$ was reduced in both DMF treatment groups compared to that in the CMC group. While TNF- $\alpha$, IL- 6 , and IFN- $\gamma$ were all significantly reduced (Fig. 6b, $p<0.05$ ), the levels of IL- $1 \alpha$ were not reliably different from those in the CMC group. By contrast, the levels of the anti-inflammatory cytokines IL-4 and IL-10 were upregulated. However, only levels of IL-4 were significantly greater compared to the CMC group (Fig. 6b, $p<0.05$ ); large variability in IL-10 levels among the groups apparently reduced the statistical power to detect a reliable difference.

We confirmed these changes in cytokine production in DMF-treated MNCs by using RT-PCR of splenocyte samples. As shown in Fig. $6 c-h$, IFN- $\gamma$, TNF- $\alpha$, IL-6, and IL-17 mRNA levels were greatly reduced in DMF-treated groups compared to those in the CMC group (Fig. $6 \mathrm{c}-\mathrm{f}, p<0.05$ ), while IL-4 and IL-10 mRNA levels increased significantly (Fig. 6g, h, $p<0.05$ ).

\section{Discussion}

In the present study, DMF therapy suppressed the effects of EAN, manifested overtly in a delay of clinical symptom onset and in a reduction in the severity of paralysis. These beneficial overt effects were associated with reduced inflammation and demyelination of the sciatic nerves. We also observed a decrease in the often-damaging M1 macrophage phenotype and an increase in the M2 phenotype in both sciatic nerves and spleens of DMF-treated rats and in vitro. In addition, the level of $\mathrm{Nrf} 2$ and $\mathrm{HO}-1$ increased significantly in DMF-treated groups. Furthermore, DMF improved the inflammatory environment by depressing the level of pro-inflammatory cytokines and increasing the level of anti-inflammatory cytokines.

Assessing neurological symptoms through clinical scores, we observed that DMF greatly improved EAN symptoms by reducing paralysis severity, delaying the onset of the first signs of EAN, decreasing the peak clinical score, and reducing motor deficits. EMG studies revealed that DMF 
generally protected nerves from EAN-induced peripheral nerve injury by improving MNCV and amplitudes of CMAPs and by reducing the distal motor latency compared to the CMC group. Histopathologically, EAN is characterized by inflammatory cell infiltration and nerve demyelination. In the present study, both preventative and therapeutic DMF treatment significantly suppressed the accumulation of inflammatory cell infiltration and demyelination when compared to the CMC group. Taken together, these results demonstrate that DMF had beneficial effects in EAN rats.

Macrophages are broadly divided into two groups: classically activated (M1) and alternatively activated (M2) macrophages [28]. M1-type macrophages release cytokines that inhibit the proliferation of surrounding cells and damage contiguous tissue, whereas M2-type macrophages release cytokines that promote the proliferation of contiguous cells and tissue repair [10]. Furthermore, alternatively activated M2 macrophages play an immunomodulatory role in EAN $[29,30]$, leading to a favorable outcome. Consistent with the previous findings, the present study showed that the ameliorated outcome of EAN rats was associated with the polarization of macrophages toward the M2 phenotype. There were many more M2-type and fewer M1-type macrophages in the sciatic nerves of DMF-treated EAN rats. Besides, flow cytometry results showed that splenic macrophages in DMF-treated EAN rats polarized toward the M2 type. This finding was also consistent with in vitro findings showing that DMF application in the culture bath directly affected macrophages differentiation. Indeed, treating LPSstimulated macrophages with DMF significantly reduced mRNA expression of inflammatory molecules, such as iNOS and TNF- $\alpha$, and induced mRNA expression of antiinflammatory cytokines, such as IL-10. These data supported the idea that DMF improved the outcome of EAN by promoting M2 type differentiation.

Based on previous mechanistic studies with cell culture models and MS, it was proposed that DMF's therapeutic mechanism is mainly the activation of Nrf2 [19]. Evidences show that Nrf2 is beneficial for other autoimmune and inflammatory diseases as well [31-35]. Corroborating these studies, our study revealed that in EAN, DMF upregulated Nrf2 in vivo. It is reported that Nrf2 exerts anti-inflammatory and anti-oxidation effects through initiating the transcription of a range of downstream genes, mainly including NQO1 and HO-1 [36]. HO-1 participates in a critical protective mechanism that is activated during cellular stress [37] and is regarded as an adaptive cellular response against inflammatory responses and oxidative injury [38]. In EAN, HO-1 is predominantly expressed starting 11 days after immunization, with local expression persisting long after neurologic signs disappear [39]. Consistent with the findings from the previous study, we observed that $\mathrm{HO}-1$ expression is detectable during the peak phase of spontaneous EAN. Furthermore, in DMFtreated EAN rats, both the production of $\mathrm{HO}-1$ and Nrf2 were much greater than that in the control group. Considering HO-1 induction can affect macrophage polarization toward the M2 phenotype in vivo and ex vivo [22, 40], it is reasonable to hypothesize that DMF induces macrophages to polarize toward the M2 type by upregulating the level of HO-1 and Nrf2. Therefore, we further explored the expression of Nrf2 in macrophages, and result showed that treating EAN rats with DMF can upregulate its expression in macrophages. Besides, it was proposed that in mice and humans, mechanisms underlying DMF's protective effects in inflammatory autoimmune diseases also involved the inhibition of Th1/Th17 cells and induction of Th2 cells [41]. The authors attributed the polarization of $\mathrm{T}$ cells to the induction of HO-1 and thus the increased level of type II dendritic cells [41]. Therefore, the effects seen in present study on macrophages in vivo could also be secondary due to dendritic cell-mediated immune regulation. Additional in vitro investigations are needed to confirm the effects of DMF on the expression of HO-1 in macrophages and to clarify the role of $\mathrm{HO}-1$ and dendritic cells in the polarization of macrophages in EAN.

DMF has been demonstrated to reduce inflammation in autoimmune and inflammatory animal models $[23,24,33$, 41-44]. In accordance with previous reports, the present study showed that DMF reduced the inflammatory response in EAN rats and favored the outcomes. DMF reduced the mRNA expression of IFN- $\gamma$, TNF- $\alpha$, IL- 6 , and IL-17 and upregulated the mRNA expression of IL- 4 and IL-10 in the spleens of EAN rats. In addition, the same trend in cytokine profile was observed in the ELISA analysis of the supernatants of cultured spleen MNCs. These data revealed that the improvement of inflammatory environment by DMF was a basis for the protective effect in EAN. Although these data were obtained from spleen samples and may not exactly represent changes in peripheral nerves in situ, as they were obtained from animals after in vivo administration of DMF, they may nonetheless provide mechanistic insight into the physiological activities underpinning this potentially valuable therapeutic and shed light on the positive clinical outcome we observed.

\section{Conclusions}

We have demonstrated that DMF promotes EAN amelioration through a Nrf2/HO-1-mediated phenotypic shift in macrophages. The anti-inflammatory effects of DMF also improve the environmental milieu in immune organ and indirectly exert neuroprotective effects.

\section{Additional file}

Additional file 1: Figure S1. DMF promotes M2 macrophage polarization in EAN. DMF was administered orally to EAN rats ( $n=6$ in each group) from 
day 1 to day 16 or day 7 to day 16 post-immunization for preventative or therapeutic treatment, respectively. On day 16 post-immunization, sciatic nerves of each group of rats were harvested for fluorescence immunohistochemistry to analyze the proportion of $\mathrm{M} 1$ macrophages and M2 macrophages in EAN rats after DMF treatment paradigms. (A) Fluorescence photomicrographs showing $\mathrm{M} 1$ and $\mathrm{M} 2$ macrophages in the sciatic nerves of EAN rats. Tissue sections were immunofluorescence stained for markers iNOS of M1, Arg 1 of M2 macrophages, and Iba1 of general macrophages as indicated. Scale bar is $50 \mu \mathrm{m}$. (B) Quantitation of immunohistochemistry. Counts per square millimeter of $\mathrm{Iba}^{+} / \mathrm{iNOS}^{+}$ cells (M1 phenotype) in the sciatic nerve showed that DMF reduced the number of M1 macrophages in both DMF treatment paradigms. (C) Counts per square millimeter of $\mathrm{Iba}^{+} / \mathrm{Arg} 1^{+}$cells (M2 phenotype) showed that DMF-treated groups increased the number of M2 macrophages in both DMF treatment paradigms compared to the CMC group $(p<0.05)$. (DOCX $399 \mathrm{~kb})$

\section{Abbreviations}

AUC: area under the curve; CMAP: compound muscle action potential; CMC: carboxy methyl cellulose; DMF: dimethyl fumarate; EAN: experimental autoimmune neuritis; EMG: electromyography; GBS: Guillain-Barré syndrome; HO-1: hemoxygenase-1; LFB: luxol fast blue; MNC: mononuclear cell; MNCV: motor nerve conduction velocity; MTS: 3-(4,5-dimethylthiazol-2-yl)-5(3-carboxymethoxyphenyl)-2-(4-sulfophenyl)-2H-tetrazolium; TNF-a: tumor necrosis factor a; Nrf2: nuclear factor erythroid-derived 2-related factor 2; PNS: peripheral nervous system.

\section{Competing interests}

The authors declare that they have no competing interests.

\section{Authors' contributions}

$\mathrm{RH}$ carried out the general studies and drafted the manuscript. JX participated in the design of the study and performed the statistical analysis. $\mathrm{HZ}$ participated in the design of the study and performed the electrophysiological studies. JH conceived of the study and participated in its design and coordination, did the general supervision of the research group, and helped to draft the manuscript. All authors read and approved the final manuscript.

\section{Acknowledgements}

This work was financially supported by the National Natural Science Foundation of China $(81322018,81273287$, and 81100887 to JWH); the Program for New Century Excellent Talents in University of China (NCET 111067 to JWH); and the Key Project of Natural Science Foundation of Tianjin Province (12JCZDJC24200 to JWH).

Received: 7 December 2015 Accepted: 21 April 2016

Published online: 03 May 2016

\section{References}

1. Yuki N, Hartung HP. Guillain-Barre syndrome. N Engl J Med. 2012;366: 2294-304.

2. Hughes RA, Swan AV, Raphael JC, Annane D, van Koningsveld R, van Doorn PA. Immunotherapy for Guillain-Barre syndrome: a systematic review. Brain. 2007;130:2245-57.

3. Hughes RA, Cornblath DR. Guillain-Barre syndrome. Lancet. 2005;366: 1653-66.

4. Zhang HL, Zheng XY, Zhu J. Th1/Th2/Th17/Treg cytokines in Guillain-Barre syndrome and experimental autoimmune neuritis. Cytokine Growth Factor Rev. 2013;24:443-53.

5. Zhu J, Mix E, Link H. Cytokine production and the pathogenesis of experimental autoimmune neuritis and Guillain-Barre syndrome. J Neuroimmunol. 1998;84:40-52.

6. Sica A, Mantovani A. Macrophage plasticity and polarization: in vivo veritas. J Clin Invest. 2012;122:787-95.

7. Biswas SK, Mantovani A. Macrophage plasticity and interaction with lymphocyte subsets: cancer as a paradigm. Nat Immunol. 2010;11:889-96.

8. Mosser DM, Edwards JP. Exploring the full spectrum of macrophage activation. Nat Rev Immunol. 2008;8:958-69.

9. Hartung HP, Schafer B, Heininger K, Stoll G, Toyka KV. The role of macrophages and eicosanoids in the pathogenesis of experimental allergic neuritis. Serial clinical, electrophysiological, biochemical and morphological observations. Brain. 1988;111(Pt 5):1039-59.

10. Mantovani A, Biswas SK, Galdiero MR, Sica A, Locati M. Macrophage plasticity and polarization in tissue repair and remodelling. J Pathol. 2013;229:176-85.

11. Kiefer R, Kieseier BC, Stoll G, Hartung HP. The role of macrophages in immunemediated damage to the peripheral nervous system. Prog Neurobiol. 2001;64: 109-27

12. Wynn TA, Chawla A, Pollard JW. Macrophage biology in development, homeostasis and disease. Nature. 2013;496:445-55.

13. Papadopoulou A, D'Souza M, Kappos L, Yaldizli O. Dimethyl fumarate for multiple sclerosis. Expert Opin Investig Drugs. 2010;19:1603-12.

14. Lee DH, Stangel M, Gold R, Linker RA. The fumaric acid ester BG-12: a new option in MS therapy. Expert Rev Neurother. 2013;13:951-8.

15. Pitarokoili K, Ambrosius B, Meyer D, Schrewe L, Gold R. Dimethyl fumarate ameliorates Lewis rat experimental autoimmune neuritis and mediates axonal protection. PLoS One. 2015;10:e0143416.

16. Kappos L, Gold R, Miller DH, Macmanus DG, Havrdova E, Limmroth V, Polman $\mathrm{CH}$, Schmierer $\mathrm{K}$, Yousry TA, Yang M, et al. Efficacy and safety of oral fumarate in patients with relapsing-remitting multiple sclerosis: a multicentre, randomised, double-blind, placebo-controlled phase IIb study. Lancet. 2008;372:1463-72

17. Fox RJ, Miller DH, Phillips JT, Hutchinson M, Havrdova E, Kita M, Yang M, Raghupathi K, Novas M, Sweetser MT, et al. Placebo-controlled phase 3 study of oral BG-12 or glatiramer in multiple sclerosis. N Engl J Med. 2012; 367:1087-97.

18. Bomprezzi R. Dimethyl fumarate in the treatment of relapsing-remitting multiple sclerosis: an overview. Ther Adv Neurol Disord. 2015:8:20-30.

19. Linker RA, Lee DH, Ryan S, van Dam AM, Conrad R, Bista P, Zeng W, Hronowsky X, Buko A, Chollate S, et al. Fumaric acid esters exert neuroprotective effects in neuroinflammation via activation of the Nrf2 antioxidant pathway. Brain. 2011;134:678-92

20. Jung KA, Kwak MK. The Nrf2 system as a potential target for the development of indirect antioxidants. Molecules. 2010;15:7266-91.

21. Paine A, Eiz-Vesper B, Blasczyk R, Immenschuh S. Signaling to heme oxygenase-1 and its anti-inflammatory therapeutic potential. Biochem Pharmacol. 2010:80:1895-903.

22. Naito $Y$, Takagi T, Higashimura $Y$. Heme oxygenase-1 and anti-inflammatory M2 macrophages. Arch Biochem Biophys. 2014;564:83-8.

23. Robles L, Vaziri ND, Li S, Masuda Y, Takasu C, Takasu M, Vo K, Farzaneh SH, Stamos MJ, Ichii H. Dimethyl fumarate protects pancreatic islet cells and non-endocrine tissue in L-arginine-induced chronic pancreatitis. PLoS One. 2014;9:e107111.

24. Robles L, Vaziri ND, Li S, Takasu C, Masuda Y, Vo K, Farzaneh SH, Stamos MJ, Ichii $\mathrm{H}$. Dimethyl fumarate ameliorates acute pancreatitis in rodent. Pancreas. 2015:44:441-7.

25. Sarkey JP, Richards MP, Stubbs Jr EB. Lovastatin attenuates nerve injury in an animal model of Guillain-Barre syndrome. J Neurochem. 2007;100:1265-77.

26. Xiao J, Zhai H, Yao Y, Wang C, Jiang W, Zhang C, Simard AR, Zhang R, Hao J. Chrysin attenuates experimental autoimmune neuritis by suppressing immuno-inflammatory responses. Neuroscience. 2014;262:156-64.

27. Zhang CJ, Zhai H, Yan Y, Hao J, Li MS, Jin WN, Su N, Vollmer TL, Shi FD. Glatiramer acetate ameliorates experimental autoimmune neuritis. Immunol Cell Biol. 2014:92:164-9.

28. Gordon S. Alternative activation of macrophages. Nat Rev Immunol. 2003;3:23-35.

29. Zhang Z, Zhang ZY, Schluesener HJ. Compound A, a plant origin ligand of glucocorticoid receptors, increases regulatory T cells and M2 macrophages to attenuate experimental autoimmune neuritis with reduced side effects. J Immunol. 2009;183:3081-91.

30. Zhang HL, Hassan MY, Zheng XY, Azimullah S, Quezada HC, Amir N, Elwasila M, Mix E, Adem A, Zhu J. Attenuated EAN in TNF-alpha deficient mice is associated with an altered balance of M1/M2 macrophages. PLoS One. 2012;7:e38157.

31. Innamorato NG, Rojo Al, Garcia-Yague AJ, Yamamoto M, de Ceballos ML, Cuadrado A. The transcription factor Nrf2 is a therapeutic target against brain inflammation. J Immunol. 2008;181:680-9.

32. Zhao X, Sun G, Zhang J, Strong R, Dash PK, Kan YW, Grotta JC, Aronowski J. Transcription factor Nrf2 protects the brain from damage produced by intracerebral hemorrhage. Stroke. 2007:38:3280-6.

33. Zhao X, Sun G, Zhang J, Ting SM, Gonzales N, Aronowski J. Dimethyl fumarate protects brain from damage produced by intracerebral hemorrhage by mechanism involving Nrf2. Stroke. 2015;46:1923-8. 
34. Lee JM, Johnson JA. An important role of Nrf2-ARE pathway in the cellular defense mechanism. J Biochem Mol Biol. 2004;37:139-43.

35. Lee DH, Gold R, Linker RA. Mechanisms of oxidative damage in multiple sclerosis and neurodegenerative diseases: therapeutic modulation via fumaric acid esters. Int J Mol Sci. 2012;13:11783-803.

36. Yang $Y$, Jiang $S$, Yan J, Li Y, Xin Z, Lin Y, Qu Y. An overview of the molecular mechanisms and novel roles of $\mathrm{Nrf} 2$ in neurodegenerative disorders. Cytokine Growth Factor Rev. 2015;26:47-57.

37. Ryter SW, Alam J, Choi AM. Heme oxygenase-1/carbon monoxide: from basic science to therapeutic applications. Physiol Rev. 2006;86:583-650.

38. Ryter SW, Choi AM. Heme oxygenase-1/carbon monoxide: from metabolism to molecular therapy. Am J Respir Cell Mol Biol. 2009;41:251-60.

39. Schluesener HJ, Seid K. Heme oxygenase-1 in lesions of rat experimental autoimmune encephalomyelitis and neuritis. J Neuroimmunol. 2000;110:114-20.

40. Mandal P, Pratt BT, Barnes M, McMullen MR, Nagy LE. Molecular mechanism for adiponectin-dependent M2 macrophage polarization: link between the metabolic and innate immune activity of full-length adiponectin. J Biol Chem. 2011;286:13460-9.

41. Ghoreschi K, Bruck J, Kellerer C, Deng C, Peng H, Rothfuss O, Hussain RZ, Gocke AR, Respa A, Glocova I, et al. Fumarates improve psoriasis and multiple sclerosis by inducing type II dendritic cells. J Exp Med. 2011;208:2291-303.

42. Schilling S, Goelz S, Linker R, Luehder F, Gold R. Fumaric acid esters are effective in chronic experimental autoimmune encephalomyelitis and suppress macrophage infiltration. Clin Exp Immunol. 2006;145:101-7.

43. Chen H, Assmann JC, Krenz A, Rahman M, Grimm M, Karsten CM, Kohl J, Offermanns S, Wettschureck N, Schwaninger M. Hydroxycarboxylic acid receptor 2 mediates dimethyl fumarate's protective effect in EAE. J Clin Invest. 2014;124:2188-92.

44. Milenkovic M, Arsenovic-Ranin N, Vucicevic D, Bufan B, Jancic I, Stojic-Vukanic $Z$. Beneficial effects of dimethyl fumarate on experimental autoimmune myocarditis. Arch Med Res. 2008;39:639-46.

\section{Submit your next manuscript to BioMed Central and we will help you at every step:}

- We accept pre-submission inquiries

- Our selector tool helps you to find the most relevant journal

- We provide round the clock customer support

- Convenient online submission

- Thorough peer review

- Inclusion in PubMed and all major indexing services

- Maximum visibility for your research

Submit your manuscript at www.biomedcentral.com/submit 